\title{
Dynamic metabolic changes measured by indirect calorimetry during the early phase of septic shock: a prospective observational pilot study
}

\author{
Emilie Occhiali $\mathbb{D}^{1 凶}$, Maximilien Urli ${ }^{2}$, Thibaut Pressat-Laffouilhère ${ }^{3}$, Najate Achamrah ${ }^{4}$, Benoit Veber ${ }^{1}$ and Thomas Clavier ${ }^{1}$
}

(c) The Author(s), under exclusive licence to Springer Nature Limited 2021

BACKGROUND AND AIMS: Energy metabolism (energy deficit, substrate consumption) in the early phase of septic shock is not clearly understood. The objective of this study was to describe its evolution using indirect calorimetry.

METHODS: Prospective observational pilot study including ventilated adult patients with septic shock admitted in a surgical intensive care unit (ICU). Metabolic data were collected using the COSMED Q-NRG $+{ }^{\circledR}$ calorimeter: carbon dioxide production (VCO2), oxygen consumption (VO2), resting energy expenditure (REE), respiratory quotient (RQ) and the rate of substrate utilization (proteins, lipids, and carbohydrates). The main criterion was the evolution of the energy deficit (dE) from D1 to D6.

RESULTS: In total, 34 patients were included and 15 patients (age: $57.6 \pm 12.8$ years; Simplified Acute Physiology Score II: $52 \pm 11$ ) were eligible for final analysis. Time for initiation of nutritional support was $2.5 \pm 1.5$ days. The dE improved during the study period (slope $=2.9[1.8 ; 4.2] ; p<0.001)$. The REE remained stable during the first week with no significant variation (slope $=-0.16[-1.49$; $0.79] ; p=0.78$ ). The $\mathrm{RQ}$ remained stable overall (slope $=0.01[0.00 ; 0.03] ; p=0.10$ ). The substrates utilization significantly changed at $\mathrm{D} 3$ in favor of protein consumption (slope $=6.50[4.44 ; 8.85] ; p<0.001$ ) with an overall significant decrease in the consumption of non-protein substrates.

CONCLUSION: Energy deficit improved while REE and RQ remained relatively stable during the first week of ICU stay. The significance of the variations of substrates consumption was unclear. These preliminary results should be further explored with larger studies.

European Journal of Clinical Nutrition (2022) 76:693-697; https://doi.org/10.1038/s41430-021-01012-2

\section{INTRODUCTION}

Worldwide, sepsis is one of the major causes of mortality in intensive care units (ICUs). Septic shock accounts for $11 \%$ of ICU admissions [1] and is the most frequent cause of shock (62\%) [2].

The nutritional needs of septic critically ill patients are modified compared to those of the healthy population, particularly due to major protein catabolism [3-5]. However, there is no consensus yet on the best nutritional management strategy to observe in these patients (timing of nutrition start, enteral or parenteral nutrition, insulin therapy...) $[5,6]$. The lack of knowledge concerning the evolution of energy metabolism during the early phase of sepsis probably explains, at least in part, our inability to propose a nutritional strategy with a beneficial impact on morbidity or mortality.

Undernutrition is associated with a prolonged duration of mechanical ventilation, an over-risk of nosocomial infection, a longer length of stay and a mortality excess [7]. On the other hand, overfeeding would induce several complications: increase of respiratory work (linked to the raise of carbon dioxide production (VCO2)), hepatic steatosis and positive fluid balance [8]. Basal energy metabolism, diet-induced thermogenesis, thermoregulation and activity-induced energy expenditure constitute the components of total energy expenditure [9]. But the effects of surgery, invasive treatments, mechanical ventilation, and body temperature complicate the evaluation of nutritional requirements of septic critically ill patients [10]. The need to provide energy as close as possible to patient's real needs has led to the development of complex predictive formulas in ICU [11]. However, these methods remain imprecise and controversial in the era of personalized medicine. Two methods are derived from the measurement of oxygen consumption (VO2) to assess individual energy needs: Fick's method, which is invasive and underestimates energy expenditure, and indirect calorimetry (IC), based on Haldane's equation and relying on the measurement of VCO2. IC currently represents the gold standard for metabolic assessment in ICU $[12,13]$.

\footnotetext{
${ }^{1}$ Rouen University Hospital, Department of Anesthesiology, Critical Care and Perioperative Medicine, 1 rue de Germont, F-76031 Rouen Cedex, France. ${ }^{2}$ Dieppe Hospital, Department of Anesthesiology, avenue Pasteur, F-76200 Dieppe, France. ${ }^{3}$ Rouen University Hospital, Department of Biostatistics, 1 rue de Germont, F-76031 Rouen Cedex, France. ${ }^{4}$ Rouen University Hospital, Department of Nutrition, 1 rue de Germont, F-76031 Rouen Cedex, France. ${ }^{\bowtie}$ email: emilie.occhiali@chu-rouen.fr
}

Received: 25 January 2021 Revised: 31 August 2021 Accepted: 13 September 2021

Published online: 15 October 2021 
Data are lacking regarding the dynamic metabolic changes in septic ICU patients. In this context, the main objective of this pilot study was to evaluate, using IC, the energy metabolism of patients admitted in ICU for septic shock, in the very early phase.

\section{MATERIAL AND METHODS \\ Study design and ethical considerations}

This was a prospective non-observational study, approved by a national ethics committee (ID-RCB: 2019-A00760-57) and performed in accordance with French laws and with the ethical standards laid down in the Declaration of Helsinki and its later amendments [14], carried out in an adult surgical ICU of a tertiary care hospital. The written briefing note was given to the patient's relatives who could refuse to allow data acquisition for this protocol.

\section{Inclusion and exclusion criteria}

Adult patients ( $\geq 18 \mathrm{y} / \mathrm{o})$ admitted in our surgical ICU for postoperative septic shock (according to SEPSIS-3 definition [15]) and benefiting from invasive mechanical ventilation were eligible to be included in the study.

Exclusion criteria were: pregnancy, age under $18 \mathrm{y} / \mathrm{o}$ or patient under guardianship, patient refusal, contra-indication to IC use [16, 17].

To be included in the final analysis, the patients should have met the following criteria: ICU stay of at least 6 days, a minimum of 3 IC measurements during ICU hospitalization and availability of daily nitrogen balance. Patients in whom there was a technical impossibility of carrying out reliable metabolic analysis (lack of data or questionable reliability of data) were excluded from the final analysis.

\section{Objective and endpoints}

The objective of the study was to describe the evolution of metabolism during the first week of ICU stay of patients admitted for septic shock.

The primary endpoint was the evolution of energy deficit ( $\mathrm{dE}$; difference between exogenous calorie intake and calorie target measured by IC) during the first 6 days of ICU stay. In our study, the REE corresponded to the calorie target of patients measured by indirect calorimetry, reduced by $30 \%$ as recommended by the European Society for Clinical Nutrition and Metabolism (ESPEN) during the acute phase of shock [13]. As the acute phase was considered to be from D1 to D6 in the study, the calorie target did not change from D1 to D6.

The secondary endpoints were the change of other metabolic markers: resting energy expenditure (REE), respiratory quotient (RQ), nitrogen balance, and substrate use (carbohydrates, lipids, proteins).

\section{Management of nutritional support and use of indirect calorimetry}

The nutritional support strategy is standardized in our ICU with a protocol regularly updated according to the most European recent recommendations (Supplementary File 1). At the time of this study, energy targets were still defined according to aggression phases: $20 \mathrm{kcal} / \mathrm{kg} / \mathrm{d}$ in acute phase (from D1 to D6), $25 \mathrm{kcal} / \mathrm{kg} / \mathrm{d}$ in post-acute phase and $30 \mathrm{kcal} / \mathrm{kg} / \mathrm{d}$ in postaggressive phase (usually when the patient is close to ICU discharge). During the study, ICU physicians prescribed the nutritional support blinded to the IC results. The calorimeter used in the surgical ICU is the Q-NRG + ${ }^{\circledR}$

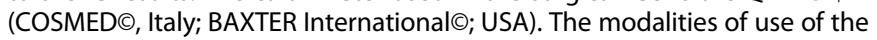
calorimeter are described in the Supplementary File 1.

\section{Data collection}

The following data were collected:

- Demographic and biological data: age, gender, body-mass index, Simplified Acute Physiology Score II (SAPS II), inflammatory markers (C-reactive protein and procalcitonin), daily urinary urea, propofol sedation;

- Characterization of septic shock: origin of sepsis, surgical diagnosis, mean norepinephrine infusion dose at D1, administration of hydrocortisone hémisuccinate (indicated in our practice for severe septic shock when the dosage of norepinephrine increases significantly despite restored blood volume);

- ICU stay and hospitalization: ICU length of stay, number of days without mechanical ventilation, ICU mortality and D28 mortality;

- Metabolic and nutritional data: $d E, R Q, R E E$, time to initiation of nutritional support, nitrogen balance, substrate utilization (carbohydrates, lipids, proteins), pre-albuminemia.

\section{Statistical analysis}

As this study is a preliminary work, strictly observational and concerning physiopathology, the number of patients to be included has not been calculated a priori.

Quantitative variable are described with means and standard deviations or $95 \%$ confidence interval and qualitative variable are described with numbers and percentages.

A piecewise linear mixed regression model with random intercept (patient) was used to quantify an abrupt change in metabolic data (Supplementary File 2). Breakpoints at D4 (for dE, RQ, REE) or D3 (for nitrogen balance and substrates consumption) were chosen after the data collection in view of the dynamic metabolic changes observed in our population. The description of the changes of the outcomes was therefore based on the analysis of tree slopes: the one taking into account the values from D1 to D6 (slope r), the one taking into account the values from D1 to D3-4 (slope $\mathrm{r} 1$ ), and the one taking into account the values from D3-4 to D6 (slope r2). Confidence intervals at 95\% were computed based on 10000 bootstrap repetitions with bias-corrected accelerated method. Last Carried Forward and Next Observation Carried Backward methods were used to impute missing data.

\section{RESULTS}

Between July 1, 2019 and July 1, 2020, 34 patients were included and 15 were included in the final analysis (Fig. 1). Their demographic characteristics are presented in Table 1. Emergency surgeries were represented by 8 postoperative peritonitis (anastomotic leakages) and 1 urosepsis (acute pyelonephritis due to calculi) that all required surgical management. The delay of initiation of nutritional support was $2.5 \pm 1.5$ days, and the energy target was reached within $4.7 \pm 2.8$ days. The routes of nutrition administration were as follows: enteral for $60 \%$ of patients $(9 / 15)$, mixed for $20 \%(3 / 15)$ and parenteral for $20 \%(3 / 15)$. None of the 15 patients received propofol sedation.

Overall, the energy deficit improved significantly during the first week $(r 1=4.8[3.10 ; 7.20], p<0.001 ; r 2=-0.5[-3.14 ; 1.93], p=$ $0.67 ; r=2.9$ [1.80;4.20], $p<0.001 ;$ Fig. 2A). The REE remained stable during the first week with no significant variation $(r 1=-1.3$ $[-3.67 ; 0.48], p=0.15 ; r 2=1.7[-0.38 ; 3.97], p=0.09 ; r=-0.2$ $[-1.49 ; 0.79], p=0.78$; Fig. $2 \mathrm{~B})$. The RQ remained overall stable,

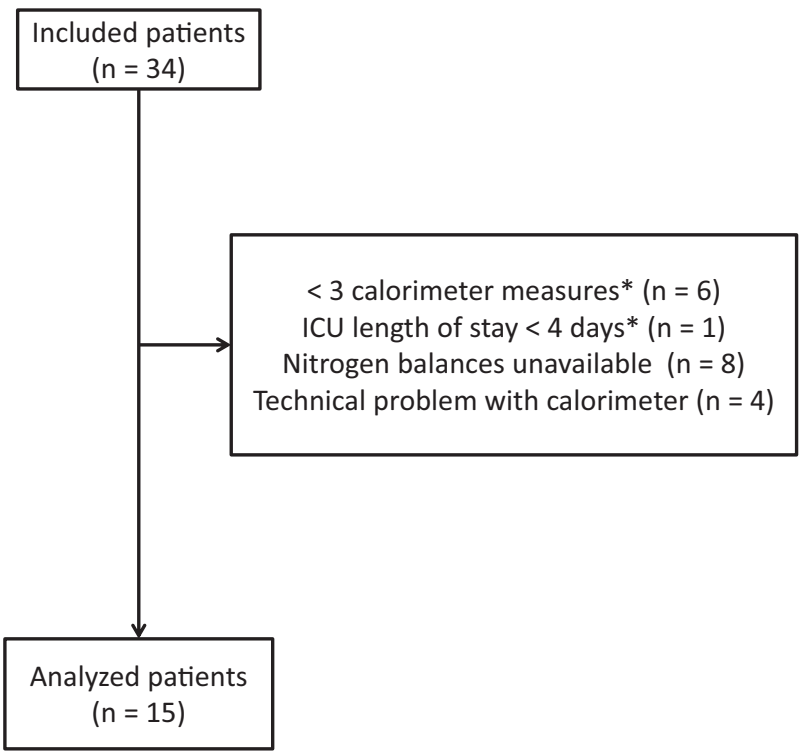

Fig. 1 Flowchart of the study. ICU intensive care unit. * due to extubation or death before day 4 . 
despite a moderate increase at the end of the period $(r 1=0.006$ $[0.03 ; 0.01], \quad p=0.55 ; \quad r 2=0.04 \quad[0.00 ; 0.09], \quad p=0.031 ; r=0.01$ $[-0.00 ; 0.03], p=0.10$; Fig. $2 \mathrm{C})$. The mean calorie intake over the first 6 days was $15.8 \pm 7.7 \mathrm{kcal} / \mathrm{kg} /$ day. The mean accumulated calorie deficit at the end of the first week for the whole cohort was $-1275 \pm 3842 \mathrm{kcal}$

Table 1. Demographic and biological characteristics.

\begin{tabular}{|c|c|}
\hline & $n=15$ patients \\
\hline Sex ratio $(M / F)$ & 0,86 \\
\hline Age (years) & $57.6 \pm 12.8$ \\
\hline $\mathrm{BMI}\left(\mathrm{kg} / \mathrm{m}^{2}\right)$ & $26.8 \pm 4.9$ \\
\hline \multicolumn{2}{|l|}{ Sepsis origin } \\
\hline Emergency surgeries & $9(60 \%)$ \\
\hline Postoperative pneumonia & $6(40 \%)$ \\
\hline SAPS II score & $52 \pm 11$ \\
\hline ICU length of stay (days) & $21.1 \pm 18.2$ \\
\hline MV free days (days) & $5.2 \pm 5.3$ \\
\hline Mean FiO2 (\%) & $40.5 \pm 11.5$ \\
\hline ICU mortality & $0 \%(0 / 15)$ \\
\hline Mortality at D28 & $6 \%(1 / 15)$ \\
\hline Mean dose of norepinephrine at D1 $(\mu \mathrm{g} / \mathrm{kg} / \mathrm{min})$ & $0.45 \pm 0.45$ \\
\hline Mean number of days of norepinephrine (days) & $3.4 \pm 1.9$ \\
\hline Patients who received HCHS & $4(26 \%)$ \\
\hline CRP D1 (mg/L) & $270 \pm 159$ \\
\hline CRP D6 (mg/L) & $123 \pm 119$ \\
\hline PCT D1 (ng/mL) & $57 \pm 83$ \\
\hline PCT D6 (ng/mL) & $1.8 \pm 2.2$ \\
\hline Prealbuminemia at $\mathrm{D} 1 \mathrm{(g/dL})$ & $0.08 \pm 0.06$ \\
\hline Prealbuminemia at $\mathrm{D} 6(\mathrm{~g} / \mathrm{dL})$ & $0.17 \pm 0.07$ \\
\hline $\begin{array}{l}\text { Number of indirect calorimetry measurements } \\
\text { (/patient) }\end{array}$ & $5 \pm 1$ \\
\hline $\begin{array}{l}\text { Initiation time of nutritional support from } \\
\text { admission (days) }\end{array}$ & $2.5 \pm 1.5$ \\
\hline
\end{tabular}

Qualitative variables are presented as absolute values and percentages: quantitative variables are presented as means and standard deviations. $B M I$ body mass index, $D$ day, $C R P$ C-reactive protein, $\mathrm{FiO}_{2}$, inspired fraction in oxygen, $H C H S$ hydrocortisone hemisuccinate, ICU intensive care unit, $M V$ mechanical ventilation, PCT procalcitonin, SAPS II simplified acute physiology score II.
Daily nitrogen inputs and losses during the first six days are shown in the Supplementary File 3. The nitrogen balance widens during the first week especially from D3 $(r 1=-1.9[-5.48 ; 0.63]$, $p=0.14 ; r 2=-4[-6.08 ;-2.05], p<0.001 ; r=-3.2[-4.60 ;-1.96]$, $p<0.001$; Fig. 3A). The consumption of proteins increased during the first week with an acceleration from D3 $(r 1=4.1[0.24 ; 8.31]$ $p=0.04 ; r 2=8[4.34 ; 12.56] p<0.001 ; r=6.5[4.44 ; 8.85] p<0.001$ Fig. 3B). Carbohydrates consumption decreased overall due to a decrease at the beginning of the period $(r 1=-7.6[-17.61 ;-1.62]$ $p=0.01 ; r 2=0.3[-3.36 ; 4.07] p=0.89 ; r=-2.7[-5.82 ;-0.17]$ $p=0.03$; Fig. $3 C$ ). The consumption of lipids evolved in two stages with an initial stability and then a marked decrease leading to an overall decrease during the study period $(r 1=3.6[-1.73 ; 11.45]$ $p=0.24 ; \quad r 2=-8.2 \quad[-13.22 ;-4.64], \quad p=0.003 ; \quad r=-3.8$ $[-6.50 ;-1.06] p=0.008$; Fig. 3D).

\section{DISCUSSION}

We showed that the energy deficit improves significantly during the first 6 days in this cohort of patients admitted for severe septic shock. Indeed, while the REE remained stable at around $25-30 \mathrm{kcal} / \mathrm{kg} / \mathrm{d}$ - values consistent with the literature in this population $[18,19]$ - daily energy balance evolving towards zero at D6 with a trend breaking at D4. First, this evolution seems to correspond to the gradual increase in calorie intake. The mean delay to introduce nutritional support was two days, contrary to what the ICU nutritional protocol recommended (initiation at D1); despite nutrition education, some non-expert physicians may still consider nutritional support as a non-essential treatment to be initiated immediately. Second, the mean calorie intake over the 6 days is rather low. The energy target could not be reached for traditional reasons in a surgical ICU (postoperative digestive disorders requiring the discontinuation of $\mathrm{EN}$, interruption of intake due to repeat surgeries) and for more questionable but real life reasons (prescription omissions). Finally, the resolution of the inflammatory syndrome probably plays a role in the nutrient efficiency as shown by the decrease in biological markers of inflammation and the increase in prealbuminemia.

Our results seem to show a significant increase in protein substrate consumption with acceleration at D3. It was concomitant to a worsening of the nitrogen balance (probably underestimated because the nitrogen losses were based on urinary urea dosage). ESPEN Guidelines do not propose a precise temporal strategy about protein supplementation, as the literature does not reach a consensus on this point [20-22]. Acceleration in proteolysis at D3 could suggest the persistence of a significant neoglucogenesis beyond 3 days. An exhaustive analysis of protein metabolism, probably not feasible in clinical practice, coupled
A

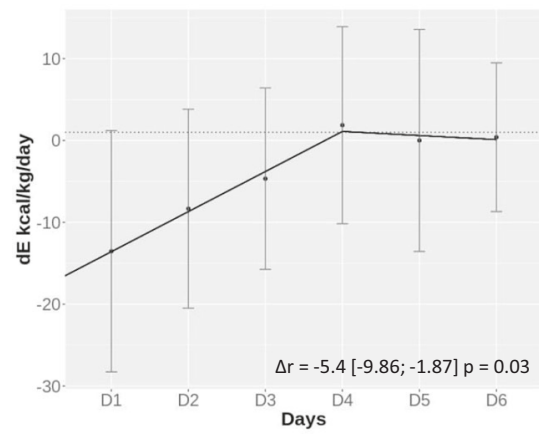

B

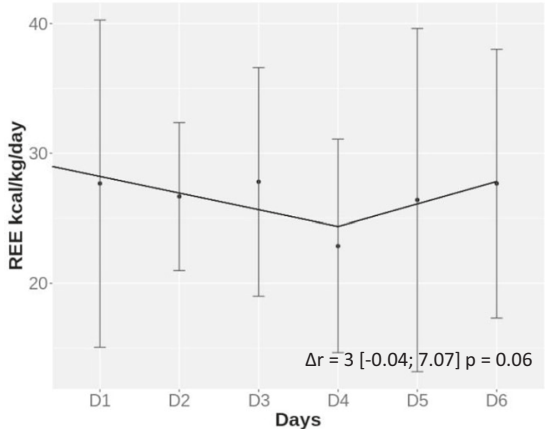

C

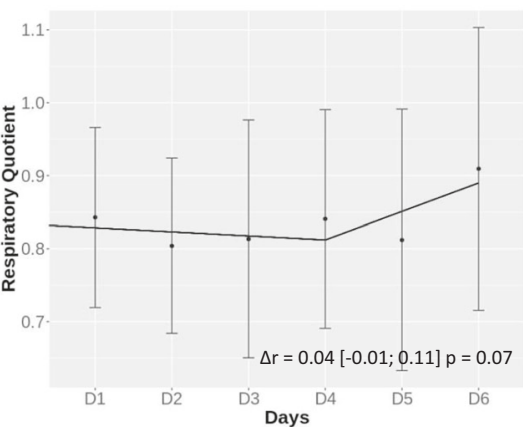

Fig. 2 Energy deficit, resting energy expenditure and respiratory quotient from day 1 to day 6. A-C Daily energy deficit (dE), daily resting energy expenditure (REE), and daily respiratory quotient from day 1 to day 6 . Values are presented as mean and standard deviations. The slopes ( $r)$ corresponding to the periods D1-D4 ( $r 1)$ and D4-D6 ( $r 2)$ are also represented on the graph. The difference between slopes $(\Delta r)$ allows the comparison of $r 1$ and $r 2$ to highlight a significant break in the evolution of metabolic data between D1-D4 and D4-D6. 
A

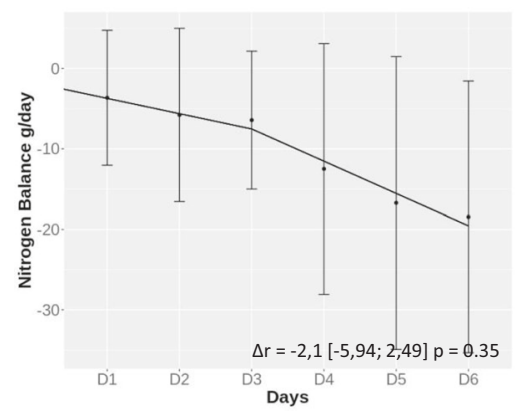

C

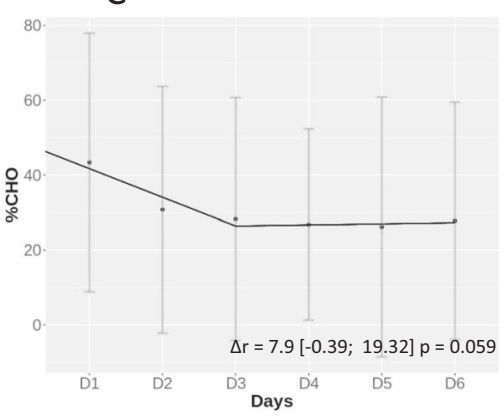

B

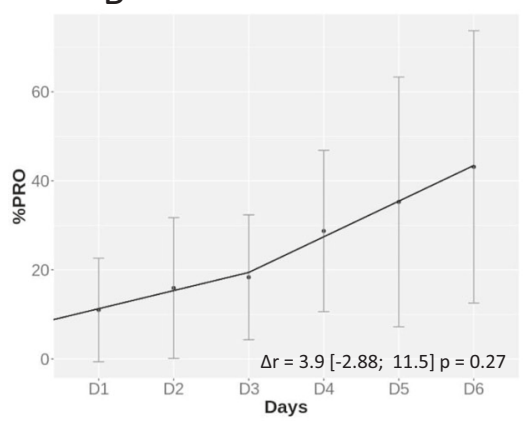

D

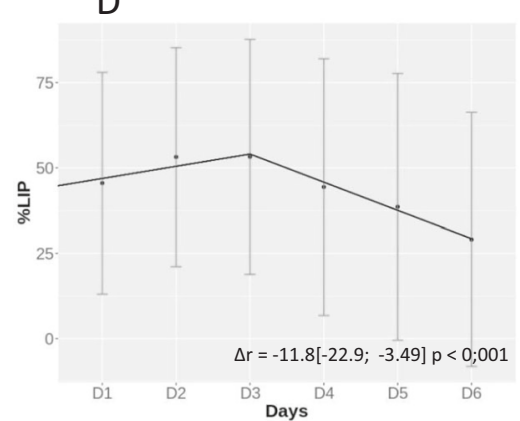

Fig. 3 Nitrogen balance and use of substrates from day 1 to day 6. A Daily nitrogen balance from day1 to day 6. B-D Daily proportion of energy expenditure related to the use of protein (\%PRO), carbohydrate (\%CHO), and lipid (\%LIP) substrates from day 1 to day 6 . Values are presented as mean and standard deviations. The slopes ( $r$ ) corresponding to the periods D1-D3 (r1) and D3-D6 (r2) are also represented on the graph. The difference between slopes $(\Delta r)$ allows the comparison of $r 1$ and $r 2$ to highlight a significant break in the evolution of metabolic data between D1-D3 and D3-D6.

with indirect calorimetry, could further explore this finding. If confirmed, an early and consistent protein intake between D1 and D3 should perhaps be considered.

With the respective changes in substrates consumption found in this study (increase in proteins consumption, decrease in lipids and carbohydrates consumption), the RQ should have remained unchanged or should have decreased. Moreover, in this septic shock context, RQ could be biased by lactate production and anaerobic metabolism. As these results cannot be easily explained, we must be cautious about drawing conclusions.

A recent meta-analysis showed that IC-guided nutritional support could have a benefit on short-term mortality [23], which supports the latest ESPEN Guidelines. Nevertheless, the difficulty of adapting the nutritional support with the help of IC is linked to the impossibility of estimating the endogenous calorie production related to neoglucogenesis during the early phase of ICU, when $50-75 \%$ of energy could be covered by glucose endogenous production through the mobilization of muscle proteins, glycogen, and lipid stores. Routine tool to evaluate the endogenous glucose production does not exist. Isotopic methods have made it possible to evaluate it at more than half of the REE during the first 3 to 4 days of metabolic aggression [24]. Providing $100 \%$ of the REE measured by IC during the acute phase, when endogenous calorie production is high, would then lead to overnutrition. IC cannot differentiate the contribution of endogenous calorie production to the overall energy deficit from the contribution of exogenous calorie intake. Then, it is difficult to know when to perform calorimetry in critically ill patient [16].

In this study, we have reported some new insights to the better understanding of metabolic changes during the early phase of septic shock. The dynamic vision is the strong point of our observation, being possible by repeating daily IC measurements and because each patient was his or her own witness. Moreover, the cohort is well representative of the severity of septic shock based on demographic characteristics.

Despite encouraging findings, the small size of the cohort is its main limitation. First, the planned 12-month inclusion period was disrupted due to two events: the COVID-19 pandemic with the transformation of our surgical ICU into a COVID medical ICU and an unscheduled maintenance of two months on the calorimeter. Second, a large number of patients admitted to our ICU for postoperative septic shock were not under mechanical ventilation or remained so for only a few hours, making inclusion impossible. Third, we had to exclude several patients from metabolic analysis because of too many missing data: impossibility to assess urinary nitrogen losses (e.g., anuric patients after inclusion) without possibility to calculate the proportions of each substrate used and patients for whom extubation or death occurred before D6. Fourth, the patients had to be in ICU, alive and mechanically ventilated at least 3 days to be analysed, creating a survivor bias. Therefore, our results cannot be extrapolated to every septic patient admitted to ICU. Another limitation is that calories provided by glucose solutions have not been taken into account; but our policy in the ICU is to limit glucose solutions perfusion as much as possible as soon as the patient receives a nutritional support. Finally, the very early metabolic evaluation by IC in this study would be questionable from the point of view of an early adaptation of the nutritional support to IC results; in that case, and according to ESPEN recommendations, measurements should have been performed from D3-D4 and beyond D6. Instead, here we wanted to emphasize the possibility of obtaining reliable metabolic profiles via IC in daily practice. Indeed, we believe that the concomitant analysis of metabolic profiles obtained by IC and that of inflammatory and insulin resistance markers could open up important fields of investigation to further personalize nutritional support. 


\section{CONCLUSION}

In this study, we have reported some new insights to the better understanding of metabolic changes during the early phase of severe septic shock, by repeating daily calorimetry measurements. Despites encouraging findings, several limits prevented us from making clear conclusions about variations in substrate consumption. Indirect calorimetry has to be used to adapt the nutritional support but it also could be used as a diagnostic tool, associated with biomarkers, to identify breakpoints in the evolution of energy metabolism and thus to personalize the nutritional management.

\section{DATA AVAILABILITY}

The raw data supporting the conclusions of this manuscript can be made available on request by the authors to any qualified researcher.

\section{REFERENCES}

1. Kaukonen K-M, Bailey M, Suzuki S, Pilcher D, Bellomo R. Mortality related to severe sepsis and septic shock among critically ill patients in Australia and New Zealand, 2000-2012. JAMA. 2014;311:1308-16.

2. Vincent J-L, De Backer D. Circulatory shock. N Engl J Med. 2013;369:1726-34.

3. Wei X, Day AG, Ouellette-kuntz H, Heyland DK. The association between nutritional adequacy and long-term outcomes in critically III patients requiring prolonged mechanical ventilation: a multicenter cohort study. Crit Care Med. 2015;13:1569-79.

4. Alberda C, Gramlich L, Jones N, Jeejeebhoy K, Day AG, Dhaliwal R, et al. The relationship between nutritional intake and clinical outcomes in critically ill patients: results of an international multicenter observational study. Intensive Care Med. 2009;35:1728-37.

5. Harvey SE, Parrott F, Harrison DA, Sadique MZ, Grieve RD, Canter RR, et al. A multicentre, randomised controlled trial comparing the clinical effectiveness and costeffectiveness of early nutritional support via the parenteral versus the enteral route in critically ill patients (CALORIES). Health Technol Assess Winch Engl. 2016;20:1-144.

6. Reignier J, Boisramé-Helms J, Brisard L, Lascarrou J-B, Ait Hssain A, Anguel N, et al. Enteral versus parenteral early nutrition in ventilated adults with shock: a randomised, controlled, multicentre, open-label, parallel-group study (NUTRIREA-2). Lancet Lond Engl. 2018;391:133-43.

7. Lefrant J-Y, Hurel D, Cano NJ, Ichai C, Preiser J-C, Tamion F. Nutrition artificielle en réanimation. Ann Fr Anesth RéAnimat. 2014;33:202-18.

8. Singer P, Hiesmayr M, Biolo G, Felbinger TW, Berger MM, Goeters C, et al. Pragmatic approach to nutrition in the ICU: Expert opinion regarding which calorie protein target. Clin Nutr. 2014;33:246-51.

9. Weekes E. Energy and nitrogen requirements in disease states. J Hum Nutr Diet. 2008;21:91-2.

10. Reid CL. Nutritional requirements of surgical and critically-ill patients: do we really know what they need? Proc Nutr Soc. 2004;63:467-72.

11. Ireton-Jones $C$, Jones JD. Why use predictive equations for energy expenditure assessment? J Am Diet Assoc. 1997;97:A44.

12. Singer $P$, Singer J. Clinical guide for the use of metabolic carts: indirect calorimetry-no longer the orphan of energy estimation. Nutr Clin Pract. 2016;31:30-8.

13. Singer $P$, Blaser AR, Berger MM, Alhazzani W, Calder PC, Casaer MP, et al. ESPEN guideline on clinical nutrition in the intensive care unit. Clin Nutr. 2019;38:48-79.

14. Toulouse E, Masseguin C, Lafont B, McGurk G, Harbonn A, Roberts JA, et al. French legal approach to clinical research. Anaesth Crit Care Pain Med. 2018;37:607-14.

15. Singer M, Deutschman CS, Seymour CW, Shankar-Hari M, Annane D, Bauer M, et al. The third international consensus definitions for sepsis and septic shock (Sepsis-3). JAMA. 2016;315:801-10
16. Delsoglio M, Achamrah N, Berger MM, Pichard C. Indirect calorimetry in clinical practice. J Clin Med. 2019;8:1387.

17. Achamrah N, Delsoglio M, De Waele E, Berger MM, Pichard C. Indirect calorimetry: the 6 main issues. Clin Nutr. 2021;40:4-14.

18. Panitchote A, Thiangpak N, Hongsprabhas $P$, Hurst $C$. Energy expenditure in severe sepsis or septic shock in a Thai Medical Intensive Care Unit. Asia Pac J Clin Nutr. 2017;26:794-7.

19. Lee PS-P, Lee KL, Betts JA, Law KI. Metabolic requirement of septic shock patients before and after liberation from mechanical ventilation. JPEN J Parenter Enter Nutr. 2017;41:993-9.

20. Zusman O, Kagan I, Theilla M, Bendavid I, Cohen J, Singer P. MON-P013: early administration of protein in critically III patients improves survival: a large retrospective cohort study. Clin Nutr 2017;36:S184.

21. Weijs PJ, Looijaard WG, Beishuizen A, Girbes AR, Oudemans-van Straaten HM. Early high protein intake is associated with low mortality and energy overfeeding with high mortality in non-septic mechanically ventilated critically ill patients. Crit Care. 2014;18:701

22. Koekkoek WACK, van Setten $\mathrm{CH}$, Olthof LE, Kars JCNH, van Zanten ARH. Timing of PROTein INtake and clinical outcomes of adult critically ill patients on prolonged mechanical VENTilation: The PROTINVENT retrospective study. Clin Nutr 2019;38:883-90. 20

23. Pertzov B, Bar-Yoseph H, Menndel Y, Bendavid I, Kagan I, Glass YD, et al. The effect of indirect calorimetry guided isocaloric nutrition on mortality in critically ill patients-a systematic review and meta-analysis. Eur J Clin Nutr. 2021. https://doi. org/10.1038/s41430-021-00919-0. Epub ahead of print.

24. Berger MM, Pichard C. Feeding should be individualized in the critically ill patients. Curr Opin Crit Care. 2019;25:307-13.

\section{AUTHOR CONTRIBUTIONS}

EO was involved in the study conception and design, in acquisition of data, in statistical analysis and interpretation of data, and in manuscript draft. MU was involved in the study conception and design, in acquisition of data, and in statistical analysis and interpretation of data. TPL was involved in statistical analysis and interpretation of data, and in manuscript draft. NA and BV were involved in interpretation of data and in manuscript revision. TC was involved in statistical analysis and interpretation of data, and in manuscript draft. All authors contributed to manuscript revision, read and approved the submitted version.

\section{COMPETING INTERESTS}

The authors declare no competing interests.

\section{ADDITIONAL INFORMATION}

Supplementary information The online version contains supplementary material available at https://doi.org/10.1038/s41430-021-01012-2.

Correspondence and requests for materials should be addressed to Emilie Occhiali.

Reprints and permission information is available at http://www.nature.com/ reprints

Publisher's note Springer Nature remains neutral with regard to jurisdictional claims in published maps and institutional affiliations. 